Copyright (@ 2009 Institute of Electrical and electronics Engineers, Inc.

All Rights reserved.

Personal use of this material, including one hard copy reproduction, is permitted.

Permission to reprint, republish and/or distribute this material in whole or in part for any other purposes must be obtained from the IEEE.

For information on obtaining permission, send an e-mail message to stds-igr@ieee.org.

By choosing to view this document, you agree to all provisions of the copyright laws protecting it.

Individual documents posted on this site may carry slightly different copyright restrictions.

For specific document information, check the copyright notice at the beginning of each document. 


\section{FUZZY MODELLING OF WAGON WHEEL UNLOADING DUE TO LONGITUDINAL IMPACT FORCES}

\author{
Russel J. Stonier \\ Faculty of Informatics and Communications \\ Central Queensland University \\ Rockhampton, Queensland, 4702 \\ Australia \\ Email: r.stonier@cqu.edu.au
}

\author{
Sita Kuppa \\ Peter J. Thomas* \\ Colin Cole \\ Centre for Railway Engineering \\ Central Queensland University \\ Rockhampton, Queensland, 4702 \\ Australia \\ Email: p.thomas@cqu.edu.au
}

\section{ABSTRACT}

It this paper a fuzzy model is developed to predict wagon wheel unloading due to wagon body and bogie pitch modes in. duced by longitudinal impact forces.

Data was obtained using the wagon dynamics simulation package, VAMPIRE(B) with a wagon model typical of wagons in Australian freight service. Simulations were completed for 31 states of wagon body mass from empty to maximum load, 8 to 62 tonne and 31 states of longitudinal force were applied making 961 scenarios.

Four 2-input 1-output fuzzy systems were developed to predict wheel unloading for each axle using wagon mass and the impact force magnitude as inputs. A cooperarive co-evolutionary algorithm was used to determine a complete set fuzzy rules for this system together with defining fuzzy set definitions. The fuzzy model produced was then evaluated for its suitability as a predictive model.

\section{INTRODUCTION}

Freight train operations such as acceleration, braking and stopping practices result in dynamic interactions among individual vehicles (wagons) and also between the vehicle and the track system. The longitudinal in-train coupler forces generated by those interactions can cause wagon instability due to wheel un-

\footnotetext{
"Address all correspondence to this author.
}

loading and increases in the Load on Velocity $(L / V)$ ratio. Wheel unloading can be increased by both wagon and bogie pitch motions [1] and lateral components of longitudinal forces due to coupler angling on curves $[2,3]$. Large longitudinal forces combined with coupler angling can also adversely affect $L / V$ ratios $[2,3]$.

The focus of this paper is on wheel unloading due to pitch motions. The wagon body and bogie pitch motions considered in this paper are limited to those caused by the impact conditions allowed by coupler slack in auto coupler systems. The 'nunin' or 'run-out' forces developed can be large enough to cause dangerous levels of axle and wheel unloading [1]. The mass of the wagon dictates the type of behaviour. Empty wagons have a lower centre of mass, usually close to coupling level. Wheel unloading in empty wagons due to longitudinal impacts is primarily via bogie pitch; wagon body pitch is minimal. Conversely, in loaded wagons, with higher centre of mass, wagon body pitch is the primary mechanism. The wheel unloading response obtained is a function of longitudinal force, mass, wagon geometry and suspension design.

For rail operations, precise calculation of wheel unloading states is only of interest when levels approach and exceed standard practice limits for safe operation. While criteria differ, wheel unloading of up to $90 \%$ is often accepted for pitch and bounce modes on straight track [4]. Lower levels are set for curve operation via wheel climb criteria $[5-7]$.

Copyright (C) 2005 by ASME 
A useful description of the instability of the rail wagon can be obtained by introducing an approximation of fuzziness. A fuzzy system is capable of performing the transformation of a human knowledge base into a mathematical formula. A fuzzy logic system allows a non-linear mapping of an input data vector into a useful scalar output. In this case, it is proposed that the scenario data base, Figures 1 to 4, could be learned to produce a fuzzy knowledge base to give predictions of wagon stability. There is no a priori knowledge of the fuzzy system and it was decided to use Evolutionary Algorithms (EAs) to find a suitable fuzzy knowledge base and fuzzy set definitions.

Step-wise numerical simulation is still too computationally expensive to be used in on-line prediction systems using embedded processors. A fuzzy model can map the input space to the output (as a black box model) with low computational load. It is envisaged that the usefulness of this work will be realised in real time implementations.

\section{WAGON MODEL DETAILS}

A model typical of wagons in Australian freight service was developed in VAMPIRE(B). The wagon model consisted of eleven masses: wagon body, 2 bolsters, 4 side frames and 4 wheel sets. The bogies modelled were Ride Control type three piece bogies. Suspension springs and constant force wedge type friction dampers (snubbers) were modelled for the secondary suspension. Primary suspension and other connections were modelled as stiff springs approximating steel-on-steel connections. The wagon model included degrees of freedom for pitch rotation for wagon body, bolsters and side frames. The wagon body to bolster connection, (centre bowl) was modelled with four (A) lift-off type steel-on-steel connections. The bolster to side frame connection was modeljed with two springs and two damper elements. The two springs and two dampers are arranged fore and aft in the spring nest to allow side frame and bolster pitch. Side frame to wheel set connections were modelled as iff-off type steel-on-steel springs. -

The VAMPIREß model was not explicitly validated against service data as the intention of the study is to explore the possibilities offered by fuzzy models. However, the VAMPIRE(B) model response was tuned to give responses typical of measured data for an eighty tonne hopper wagon.

\section{DATA GENERATION AND ANALYSIS}

Simulations were completed for 31 states of wagon body mass from empty to maximum load, 8 to 62 tonne: $x_{1}=8+$ $1.8(i-1)$ for $i=1 \cdots, 31$. Longitudinal impact forces were approximated by a sinusoidal force profile applied to the wagon model. A force profile with a frequency of $5 \mathrm{~Hz}$ was selected as ant approximation of in-service impact behaviour. Further study is in progress using different force profiles. A total of 31 states

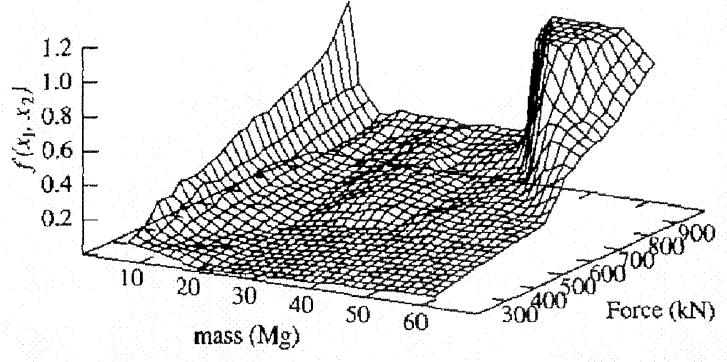

Figure 1. WHEEL UNLOADING $-Q / Q_{S}$ AXLE 1

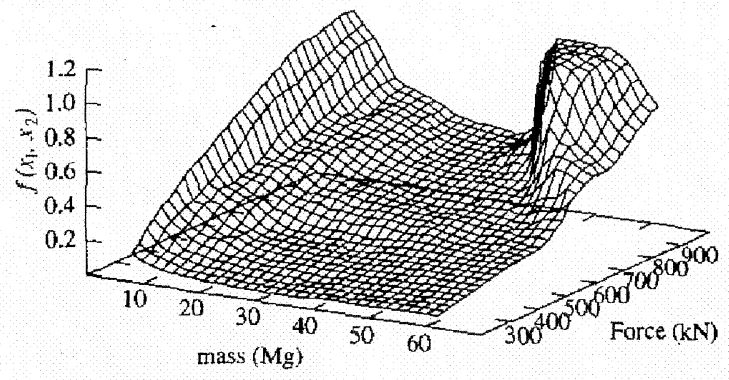

Figure 2. WHEEL UNLOADING $-Q / Q_{s}$ AXLE2

of longitudinal force were applied with magnitudes ranging from 200 to $980 \mathrm{kN}: x_{2}=200+26(i-1)$ for $i=1, \cdots, 31$. The results of these simulations were then examined and the worst case of wheel unloading in each simulation recorded. This allows the analysis to be simplified to a set of scenarios rather than a fully detailed analysis of time series response data. As these predictions would be utilised in either train driver simulations or train advisory systems it was considered adequate that only the extreme or maximum wheel unloading be predicted in each scenario. The training data set for each output of the fuzzy system therefore consisted of 961 data points as shown in Figures 1, 2, 3 , and 4 .

\section{FUZZY CONTROL SYSTEM DESIGN}

Fuxzy systems [8] are an abstraction of set theory that extends into being partially in a set and partially not (classical set theory is a subset of fuzzy set theory). The amount that an input variable is contained in a set is controlled by some function (triangular, trapezoidal, Gaussian, bezier curves, bipolar continuous, etc.). Each input variable has a domain that can be covered by several fuzzy sets. The extension principle allows the input space to become an $n$-dimensional space. A rule considers each 


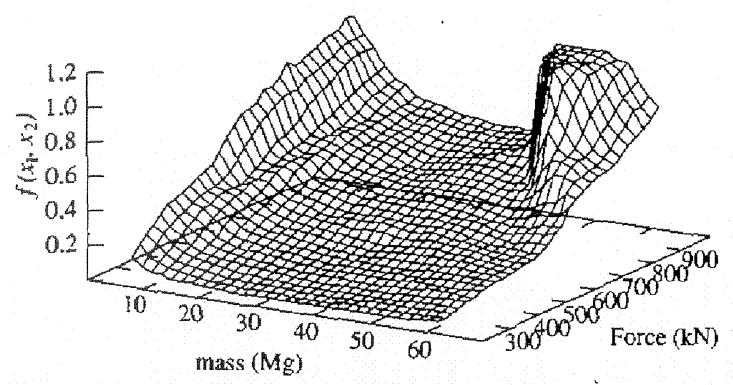

Figure 3. WHEEL UNLOADING $-Q / Q_{s}$ AXLE 3

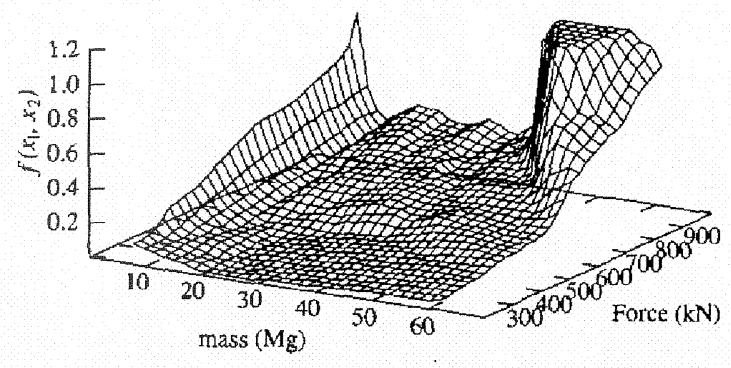

Figure 4. WHEEL UNLOADING $-Q / Q_{s}$ AXLE 4

input variable against the sets defined over the domain of that variable. Rules can also use inclusion and exclusion conditions (and, or) commonly found in logic.

An inference engine defines how sets are combined when inclusion and exclusion are used in a rule. The inclusion and exclusion logic forms are now converted to a function.

As can be imagined, several rules will 'fire' for a given input. The outputs of the rules can be combined to form a single result by using 'centre of gravity', 'cèntre average', or a 'maximum" defuzzifier.

The inputs into the fuzzy knowledge base are now variables $x_{1}$ and $x_{2}$. The output from each fuzzy system is $y_{k}$ as $-Q / Q_{s}$ for $k=1, \cdots, 4$ of axle $k$. There are seventeen linguistic membership sets defined for each variable. The seeded fuzzy set definition for input variable $x_{1}$ were completely overlapping normalised definitions: $8.0+3.375(i-1)$ for $i=1, \cdots, 17$. Similarly, the seeded fuzzy set definitions for input variable $x_{2}$ were: $200.0+48.75(i-1)$ for $i=1, \cdots, 17$.

In all there are $17^{2}=289$ rules in a complete fuzzy knowledge base for this system. In general, the $\ell^{\text {th }}$ fuzzy rule has the form:

$$
\text { If }\left(x_{1} \text { is } A_{1}^{\ell} \text { and } x_{2} \text { is } A_{2}^{\ell}\right) \text { Then }\left(y \text { is } B^{t}\right) \text {. }
$$

where $A_{k}^{\ell}, k=1,2$ are normalised fuzzy sets for input variables $x_{k}, k=1,2$, and where $B^{k}$ is a normalised fuzzy set for output variables $y$.

Given a fuzzy rule base with $M$ rules, a fuzzy controller as given in Equation 1 uses a singleton fuzzifier, Mamdani product inference engine and centre average defuzzifier to determine output variables.

$$
\hat{f}\left(x_{1}, x_{2}\right)=\frac{\sum_{k=1}^{M} \bar{Y}^{\ell}\left(\prod_{i=1}^{n} \mu_{A_{\xi}^{\xi}}\left(x_{i}\right)\right)}{\sum_{\ell=1}^{M}\left(\prod_{i=1}^{n} \mu_{A_{\xi}^{\ell}}\left(x_{i}\right)\right)}
$$

where $\bar{y}^{\ell}$ are centres of the output sets $B^{\ell}$

These values, 289 of them, are typically unknown and require determination in establishing valid mapping from input data to output data. Assuming no a priori knowledge about the system control, Evolutionary Algorithms (EA) [9] were used to search for an acceptable solution.

\section{EVOLUTIONARY LEARNING}

This paper uses a variation of the Cooperative Coevolutionary Genetic Algorithm (CCGA) presented by [10]. Instead of breaking each individual into two populations and using two Evolutionary Algorithms (EAs), the best Fuzzy Rule Base (FRB) is found in one EA and the best Fuzzy Set (FS) definition is found by a second EA. These EAs are used in a cooperative way to find a fuzzy mapping that closely resembles the simulated output from VAMPIRE(R). As this is an evolutionary technique, it should be called Cooperative Co-Evolutionary Algorithm (CCEA). A pseado-programme of the CCEA is:

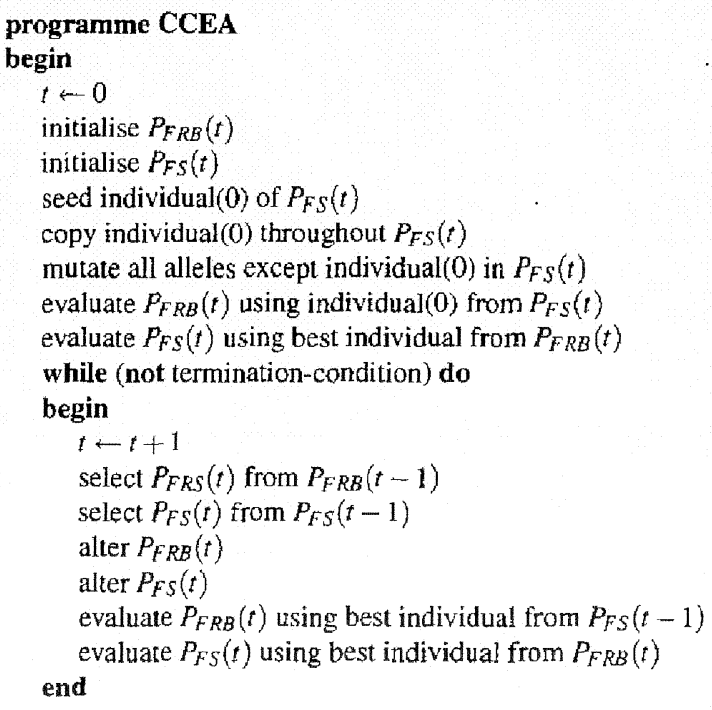

Copyright (c) 2005 by ASME 
end

The evaluation of individuals in the FRB population use the best individual from the FS population i.e. using the greedy operator approach. Similarly, the evaluation of individuals in the FS population use the best performing individual from the FRB population.

Fitness evaluation of each individual was calculated as the surn of squared errors:

$$
E=\sum_{i=1}^{961}\left(\hat{f}\left(\dot{x_{1}}, x_{2}\right)-f\left(x_{1}, x_{2}\right)\right)^{2}
$$

The value of $E$ is a good performance measure for the evolutionary algorithm, but does not portray information such as requiring the maximum relative error to be within $\pm 10 \%$. This additional requirement was met by producing a matrix of errors expressed as a percentage:

$$
E\left(x_{1}, x_{2}\right) \%=\frac{100\left(\hat{f}\left(x_{1}, x_{2}\right)-f\left(x_{1}, x_{2}\right)\right)}{f\left(x_{1}, x_{2}\right)}
$$

Convergence of the algorithm was seen at 10000 generations, however, the algorithm was terminated after $100000 \mathrm{gen}$ erations to see if any improvement could be found.

- The following discussion presents each EA in detail.

\section{FRB Evolutionary Details}

Instead of using a vector of membership sets for the output variable, each of the 289 rules has a unique membership centre $\overrightarrow{y_{k}}=$ IEEE 64 -bit floating point. Encoding of an individual in this form allows high precision numerical mapping and allows fine control for the evolutionary algorithm on the output centres.

Each output fuzzy set is represented by a Floating Point (FP) number in the interval $[0,1]$ corresponding to the fuzzy output rule. So we can uniquely represent a potential knowledge base solution as an individual string $s$ containing $M=289$ floating point numbers represented as consequents of the form:

$$
s_{F R B}=\left\{s^{1}, \cdots, s^{k}, \cdots, s^{M}\right\}
$$

The population at generation $t, P(t)=\left\{s^{n}: n=1, \cdots, N\right\}$, where $N=50$ is the number of individuals in the population. The population at the next generation $P(t+1)$ was built using a full replacement policy, tournament selection with size $n_{T}=2$, and 2D geometric crossover with probability $p_{c}=0.6$. Elitism was used by copying the best two individuals from previous population to current population. Mutation was set to a constant rate of $p_{s 3}=0.01$.
The 2D geometric crossover [11] allows the natural matrix format of data representation to be retained in crossover operations. The column or row is selected with a probability of 0.5 and follows the normal geometric crossover operation. This allows high performing row or column short defining length schemata to be retained. Otherwise, linearisation of a matrix causes column schemata to have large defining lengths and thus easily destroyed by one point geometric crossover.

The mutation uses a delta-float mutation operator that in crements/decrements $s_{k}$ by \pm 1 rand $\times\left[2^{-18}, \ldots 2^{-3}\right]$. This is achieved by using a "quick and dirty" 32-bit random number [12] twice. The first random number is used for the lower 32-bits of the 64-bit FP number. The second random number (used as the upper 32-bits of the 64-bit FP number) is masked by $0 \times 80$ FFFFFF to limit the exponential to $[0,15]$ and to retain sign of the number. An offset of $0 \times 3 E D 00000$ is added to bring the exponent in the interval $[-18,-3]$, This perturbation is then added to the allele to be mutated. There is no bounds checking on the allele and it is therefore allowed to move outside the interval $[0,1]$.

\section{FS Evolutionary Details}

Fuzzy triangular memberships definitions are encoded for each input variable $x 1$ and $x 2$ :

$$
s_{F S}=\left\{s_{1}, s_{2}\right\}
$$

where:

$$
s_{\sim}=\left\{s_{1}^{1}, s_{2}^{1}, s_{3}^{1}, \cdots, s_{1}^{k}, s_{2}^{k}, s_{3}^{k} ; \cdots, s_{1}^{N}, s_{2}^{N}, s_{3}^{N}\right\}
$$

and

$$
s_{2}=\left\{s_{1}^{1}, s_{2}^{1}, s_{3}^{1}, \cdots, s_{1}^{k}, s_{2}^{k}, s_{3}^{k}, \cdots, s_{1}^{o}, s_{2}^{o}, s_{3}^{o}\right\}
$$

where $s_{1}^{k}, s_{2}^{k}, s_{3}^{k}$ are tuples defining normalised fuzzy sets and $s_{j}^{k}$ are double precision floating point numbers.

There are $N=17$ sets defined for input variable $x l$, and $O=$ 17 sets defined for input variable $x 2$.

The population at generation $t, P(t)=\left\{s_{s S}^{n}: n=1, \cdots, N\right\}$, where $N=50$ is the number of individuals in the population. The population at the next generation $P(t+1)$ was built using a full replacement policy, toumament selection with size $n_{T}=2$, and one point crossover with probability $p_{c}=0.6$. Elitism was used by copying the best two individuals from previous population to current population. Mutation was set to a constant rate of $p_{m}=$ 0.01 .

The mutation uses the delta-float mutation operator that increments/decrements $s_{k}$ by \pm 1 .rand $\times\left[2^{-18} \cdots 2^{-3}\right]$ on $s_{1}$ or

Copyright (c) 2005 by ASME 


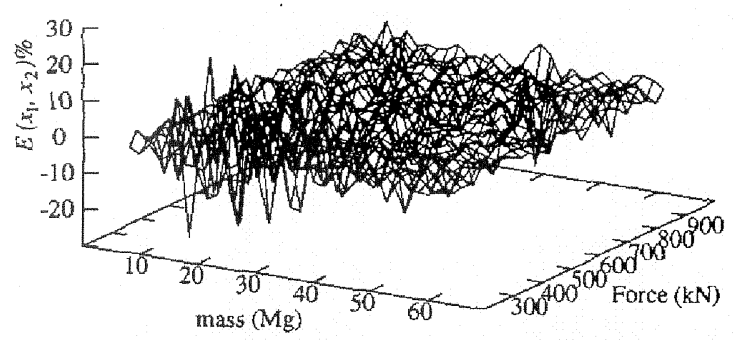

Figure 5. PERCENTAGE ERROR FOR AXLE 1

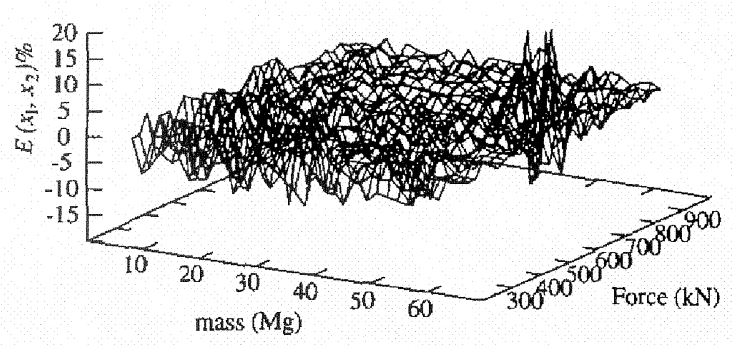

Figure 6. PERCENTAGE ERROR FOR AXLE 2

\pm 1 .rand $\times\left[2^{-17} \cdots 2^{-2}\right]$ on $s_{*}$. There is no bounds checking on the allele and it is therefore allowed to move outside the interval $[8.0,62.0]$ for $s_{1}$ and $[200.0,980.0]$ for $s_{\gamma^{\prime}}$.

\section{RESULTS}

The best fuzzy rule base string was taken from the FRB population and the best fuzzy set definition was taken from the FS population to from the fuzzy approximation in each of four cases studied.

For each of the four cases studied, the CCEA was run on eleven nodes of a Pentium cluster using $1.0 \mathrm{GHz}$ machines. Effectively, the algorithm was run eleven times for each case. All eleven runs converged to the same perfornance measure $E$ with only a very small amount of variance. The relative error $E \%$ maps of the eleven runs compared well with each other, showing a consistent convergence space of the CCEA.

Relative errors for fuzzy systems corresponding to axles one to four are shown in Figures 5 to 8 respectively. The final fitness values of the fuzzy systems developed for axles one to four are: $E=0.068,0.14,0.061$, and 0.19 respectively.

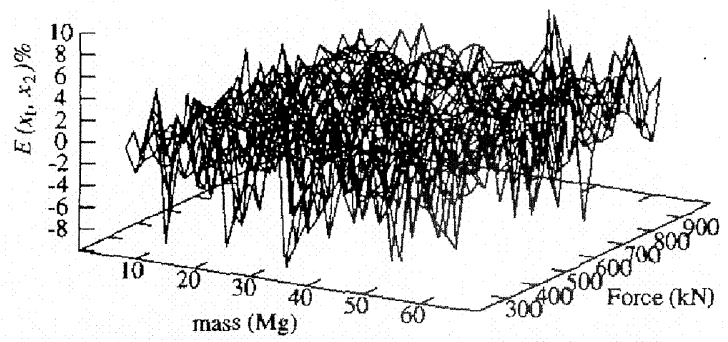

Figure 7. PERCENTAGE ERROR FOR AXLE 3

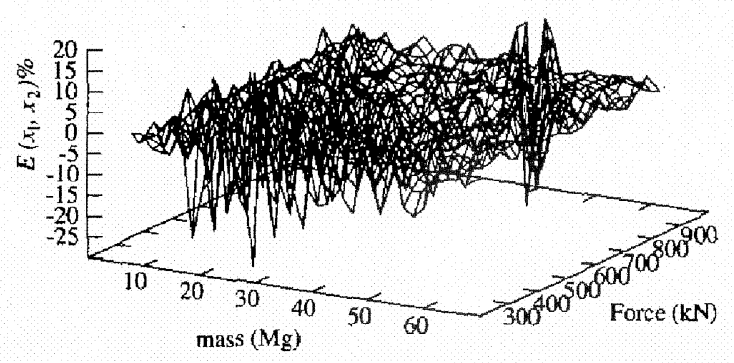

Figure 8. PERCENTAGE ERROR FOR AXLE 4

\begin{tabular}{rrr} 
Table 1. & \multicolumn{3}{c}{ RESULTS SUMMARY } \\
\hline Axle & Number & Worst \\
& $|E|>10 \%$ & $|E \%|$ \\
\hline 1 & 24 & 27.63 \\
2 & 8 & 15.81 \\
3 & 0 & 9.88 \\
4 & 58 & 26.13 \\
\hline
\end{tabular}

\section{DISCUSSION}

The resulting fuzzy system for axle one had twenty-four out of 961 input/output absolute relative errors $\left|E\left(x_{1}, x_{2}\right) \%\right|$ that exceeded ten percent. All of these errors are around the safe area of wheel unloading $-Q / Q_{s}<0.3$. The largest relative error was $E(22.4,200) \%=27.63 \%$ at $f(22.4,200)=0.02$.

The number of absolute relative errors exceeding ten percent and the worst of these errors are summarised in Table 1.

Generally, the fuzzy system mapping of input/output data for axles one to four were good with only a few unacceptable errors.

The errots are not located in any consistent area and indi-

Copyright (C) 2005 by ASME 
cate multi-objective convergence type of errors. There are many methods of reducing these types of errors that can be considered for future work.

\section{CONCLUSION}

Overall the CCEA was effective in finding a fuzzy mapping as close as possible the the original data. The resulting fuzzy system executes in approximately $35 \mu$ s on a $\mathrm{P} 42.667 \mathrm{MHz}$ machine. The execution time will be slower for an on-line embedded sys$t e m$, but is considerably faster than running numerical simulation.

The number and size of errors for axle one and four indicate that refinement of the technique is still required for precise prediction of the output of the fuzzy system. When comparing Figures 1 to 4 , it is noted that there are smoother changes in inpuvoutput data for axles two and three than for axles one and four.

The work is still at an early stage of development. As the data set was limited to a particular wagon type with a specific force profile, considerable work still remains in generalising this technique to adequately model wagon pitch responses.

Further work is being progressed in extending the database to include impact forces of larger magnitudes and over a range of different frequencies.

\section{ACKNOWLEDGMENT}

Thanks go to the Cooperative Research Centre for Railway Engineering Technologies of Australia. Also to the Centre for Rail Engineering and James Goldston Faculty of Engineering and Physical Systems at Central Queensland University. Simulations were conducted using VAMPIRE $($ AEA Technology Rail.

\section{REFERENCES}

[1] McClanachan, M., Cole, C., Roach, D. and Scown, B. 2000, Investigation of the Effect of Bogie and Wagon Pitch Associased with Longitudinal Train Dynamics, Vehicle System Dynamics, vol. 33, no, SUPPL., pp. 374-385.

[2] El-Sibaie, M. 1990, Coupler Angling under in-Train Loads: Modelling and Validation, AREA/ASME Joint Railroad Conference, Florida.

[3] El-Sibaie, M: 1993, Recent Advancements in Buff and Draft Testing Techniques, Technical Papers - IEEE/ASME Joint Railroad Conference, Publ by IEEE, Pittsburgh, PA.

[4] AAR 1993, Chapter II, in Manual of Standards and Recommended Practices, AAR, Washington.

[5] Elkins, J. and Wu, H. 1999, Investigation of Wheel Flange Climb Derailment, Association of American Railroads. Washington.
[6] Elkins, J. and Wu, H. 2000, Angle of Attack and DistanceBased Criteria for Flange Climb Derailment, In Vehicle System Dynamics 16th LAVSD Symposium - The Dynamics of Vehicles on Roads and on Tracks, Aug 30-Sep 3 1999, vol. 33, Swets en Zeitlinger B.V.,Lisse,Netherlands, Pretoria,S Afr, pp. 293-305.

[7] Elkins, J. and Wu, H. 2000, New Criteria for Flange Climb Derailment, Proceedings of the IEEE/ASME Joint Railroad Conference The 2000 Spring ASME/IEEE Joint Rail Conference,Apr 4-Apr 62000 , pp. 1-7.

[8] Wang. L. X. 1997, A Course in Fuzzy System and Control, Prentice-Hall, ISBN: 0-13-540882-2.

[9] Michalewicz, Z. 1994, Genetic Algorithms + Data Structures $=$ Evolution Programs, 2nd Edition, Springer Verlag.

[10] Potter, M. A. and de Jong, K. A. 1994, A cooperative coevolutionary approach to function optimization, in The Third Parallel Problem Solving Form Nature, Brlin, Germany: Springer-Verlag, pp. $249-257$.

[11] Young, N. and Stonier, R. 2004, Multi-dimentional Encoding to Reduce Bias in Fuzzy Knowledge-Base, Proceedings of the $7^{\text {th }}$ Asian-Pacific Complex Systems Conference (Complex 2004), Cairns, pp. 196-206, ISBN: 1-876674962.

[12] Press, W. H., Teukolsky, S. A., Vetterling, W. T., and Flannery, B. P. 1992, Numerical Recipies - The Art of Scientific Computing, Second Edition, Cambridge University Press, Cambridge. 